Appeared in: Fractals Vol. 3, No. 3, pp. 609-616 (1995), and in: Fractal Geometry and Analysis, The Mandelbrot Festschrift, Curaçao 1995, World Scientific (1996)

\title{
Fractal Geometry of Financial Time Series
}

\author{
Carl J.G. Evertsz \\ Center for Complex Systems and Visualization, University of Bremen \\ FB III, Box 330 440, D-28334 Bremen, Germany
}

\begin{abstract}
A simple quantitative measure of the self-similarity in time-series in general and in the stock market in particular is the scaling behavior of the absolute size of the jumps across lags of size $k$. A stronger form of self-similarity entails not only that this mean absolute value, but also the full distributions of lag- $k$ jumps have a scaling behavior characterized by the above Hurst exponent. In 1963 Benoit Mandelbrot showed that cotton prices have such a strong form of (distributional) self-similarity, and for the first time introduced Lévy's stable random variables in the modeling of price records. This paper discusses the analysis of the self-similarity of high-frequency DEM-USD exchange rate records and the 30 main German stock price records. Distributional selfsimilarity is found in both cases and some of its consequences are discussed.
\end{abstract}

\section{Introduction}

Self-similarity[1] in financial price records manifests itself in the virtual impossibility to distinguish a daily price record from, say, a monthly, when the axis are not labeled. Figure 1 illustrates this phenomenon for the German DAX composite index. The left figure plots the logarithm of the daily closing prices of the index over the period 1986 to 1993 . The right figure contains a 60 day daily record, a 60 week weekly record and a 60 month monthly record. It is impossible to say which of these is which (entry [2] in the reference section).

This paper discusses the quantitative analysis of the self-similarity of high-frequency DEMUSD exchange rates and that of the 30 stocks comprising the German DAX index. The statistical self-similarity observed in Figure 1 is qualitatively similar to that found in graphs of ordinary 

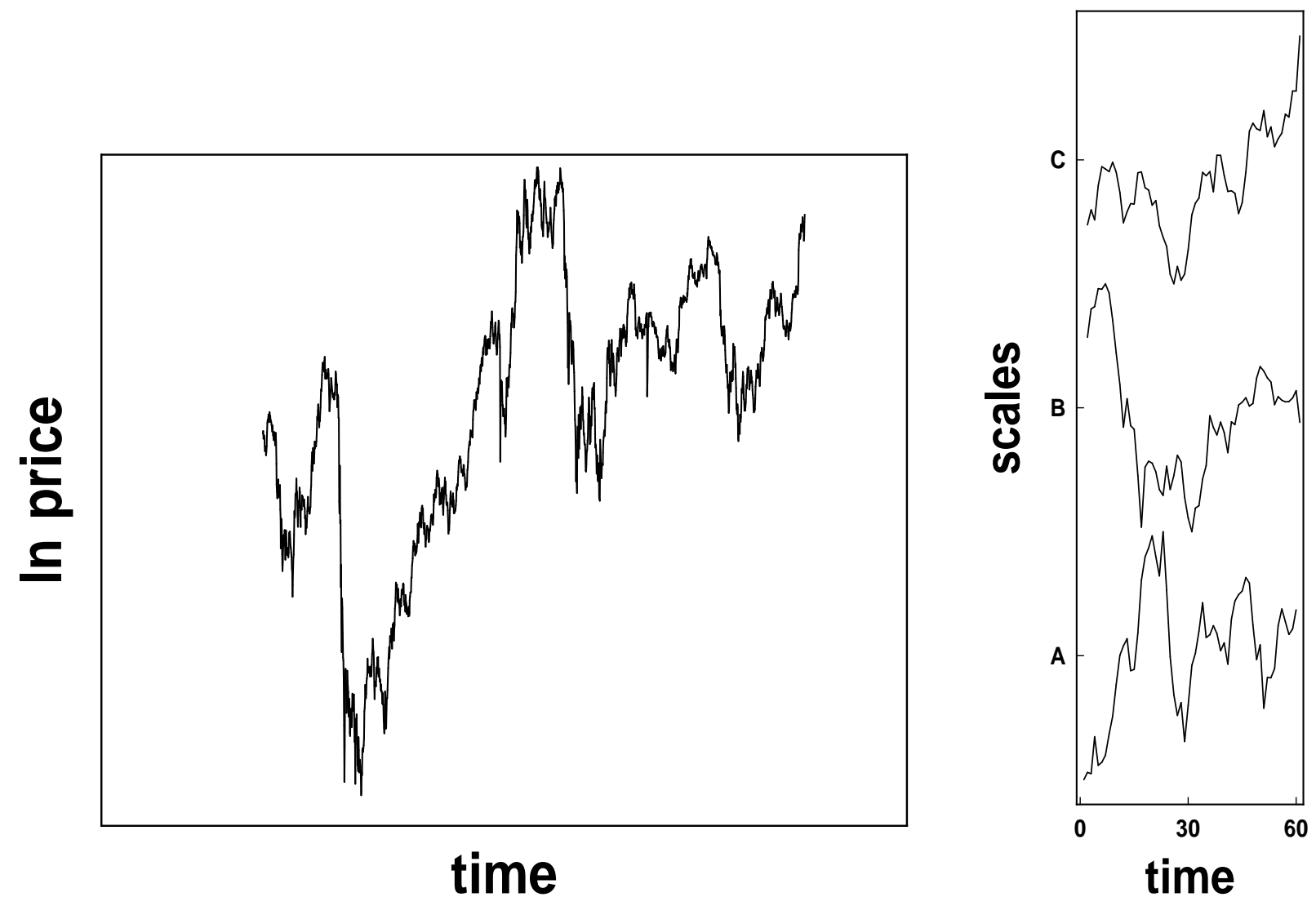

Figure 1: Left) Natural logarithm of the daily closing prices of the DAX index from November 3, 1986 till August, 4, 1993. Right) A 60 day daily price record, a 60 week weekly price record and a 60 month monthly price record. Which is which is revealed in entry [2] in the references.

Brownian motion, a theory of which was developed, and proposed as a model for stock prices by Bachelier [3, 4] in 1900. Essentially, this model is based on the assumption that price changes are independent, identically distributed, with a finite variance. In the early 1960's Mandelbrot[5] showed, that the independence of price changes and the theoretically desirable property of stability of the distributions of returns, could be reconciled with the leptokurtosis (fat tails) found in the empirical distributions of price records (see also Mirowski[6] in this volume.) Denoting the logarithm of the price at time $t$ by $Y(t)$, we find in Reference [5]:

Granted that the facts impose a revision of Bachelier's process, it would be simple indeed if one could at least preserve the convenient features of the Gaussian model that the various increments $Y(t+k)-Y(t)$, depend upon $k$ only to the extent of having different scale parameters. From all other view points, price increments over days, weeks, months and years would have the same distribution.

Under the assumption of independence, Benoit Mandelbrot was then led[5] to a Lévy stable market, where as $k$ increases the rescaled lag- $k$ returns, $k^{-1 / \alpha}(Y(t+k)-Y(t))$, would tend towards a stable random variable of index $\alpha[7]$. For Cotton prices he estimated $\alpha \approx 1.7$, which 
corresponds to a Hurst exponent $H=1 / \alpha \approx 0.59$. This scaling behavior deviates considerably from that expected in the simple Bachelier Gaussian market, where the Gaussian Central Limit theorem[8] shows that $H=\frac{1}{2}$.

\section{Hurst exponents}

Hurst exponents differing from the Gaussian $H=\frac{1}{2}$ can arise due to various causes. In the case of sums of stationary and independent random increments, values of $\frac{1}{2}<H<1$ arise when the increments have probability distributions with power-law tails $p(x) d x \sim x^{-\alpha-1}$, with $\alpha=1 / H$. In the independent case it is impossible to have $H<\frac{1}{2}$. However, when correlations are allowed, one can get all values $0<H<1$. This is exemplified by the fractional Brownian motions[9, 10]. These processes are special in the sense that, like for stable processes, also here[9]

$$
Y(t+k)-Y(t) \text { i.d. }\left(\frac{k}{k^{\prime}}\right)^{H}\left(Y\left(t+k^{\prime}\right)-Y(t)\right),
$$

that is, $k^{-H}(Y(t+k)-Y(t))$ converges to a distribution (i.d. stands for identically distributed.) However, the convergence is not to a stable distribution of index $\frac{1}{H}$, but to the Gaussian.

This paper is based on results on the self-similarity of financial time-series, reported in References [11] and [12], and places them more clearly in the above perspective. Two sets of empirical data are used. One contains the daily closing prices of the 30 DAX stocks for the period November 3, 1986, till September 7, 1992. Counting only business days, these data contain 1452 prices for each stock. Typically the records look like the left plot in Figure 1. The other data set, the USD-DEM foreign exchange rate[13], contains 1472241 bit and ask quotes occurring between October 1, 1992 and September 30, 1993. Figure 2 shows a daily and an intra-day plot of this exchange rate.

A first quantitative test for the self-similarity in a time series is to estimate the mean of the size of the jumps across time lags of size $k$, and to look for a scaling behavior of this quantity as a function of $k$. For economic time series it is customary to consider the jumps in the logarithm of a price record across lags of size $k$, since these are approximately equal to the return over such a period. For the daily DAX data, this lag is measured in units of business days. The time series studied is then $\{Y(i)\}_{t=1}^{T}$, where $Y(t)$ is the logarithm of the closing price on business day $t$. In the case of high-frequency DEM-USD exchange data, strong intra-day seasonalities and a Pareto distribution of waiting times between quotes, exclude the use of a physical time unit in which to express the lags. Using the empirical fact that the distribution of inter-quote returns is virtually independent of the physical time interval between the successive quotes[12], we therefore measure lags in terms of the numbers of quotes. For DEM-USD, $Y(t)$ is taken to be the logarithmic middle price at the $t^{\text {th }}$ quote, that is, $Y(t)=(\ln A(t)+\ln B(t)) / 2$, where $A$ and $B$ are ask and bid prices.

The estimates of the corresponding Hurst scaling exponent $H$

$$
<|Y(t+k)-Y(t)|>\sim k^{H}
$$

for various exchange rates and stocks reported in the literature, vary between $H=0.45$ and $H=0.6[5,14,15,11,12]$. Figure 3 shows the results of such an analysis $[11,12]$ for the high- 

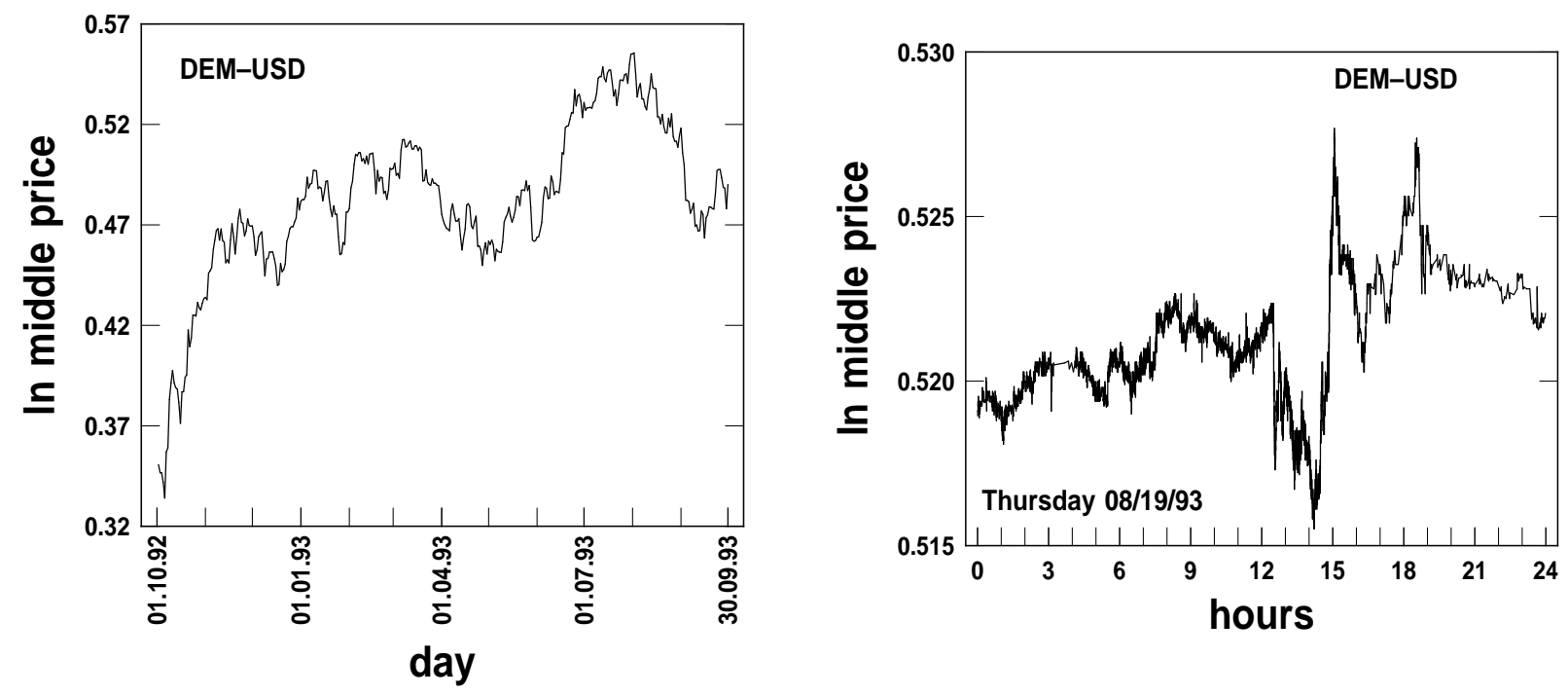

Figure 2: left) The daily logarithmic middle price record for DEM-USD. The prices plotted are those quoted closed to 3 P.M. Greenwich Mean Time. The right) plot is the intra-day logarithmic middle price record on Thursday August 19, 1993. Note the anti-correlations on the small time scales in the plot on the right. The empirical Hurst exponent is $H=0.45$ on these short time scales.
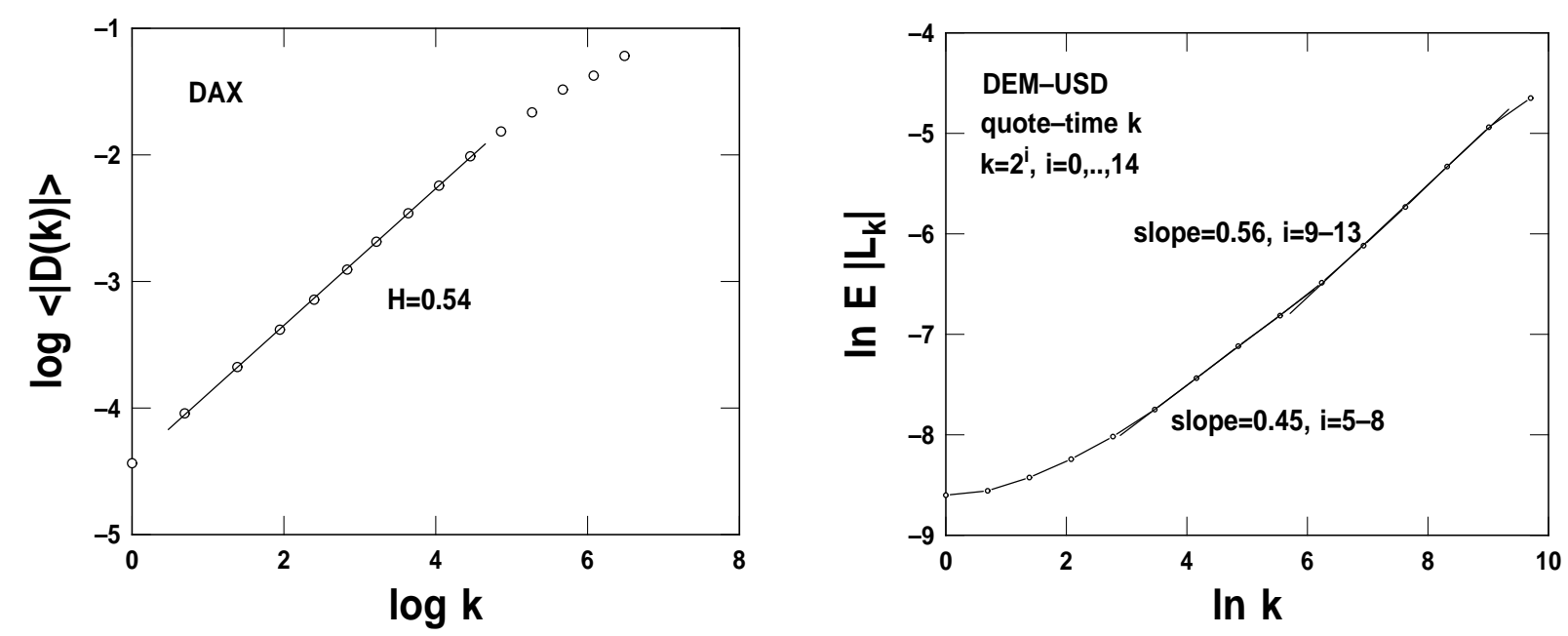

Figure 3: Log-log plot from which the Hurst exponent is estimated. left) Combining the k-day returns for all 30 DAX stocks, one finds a linear behavior for scales ranging from 1 t0 85 days. right For scales from 32 to 512 quotes the estimated Hurst exponent is 0.45 . For 512 quotes and above it is 0.56 .

frequency DEM-USD and the 30 DAX stocks. Since, all the 30 DAX stocks have approximately 
the same distribution of daily returns, we have simply combined all the lag- $k$ returns (jumps) together in one statistical ensemble. Therefore, the analysis presented here reflects a combined behavior of the 30 DAX stocks. For these combined DAX stocks, one finds a scaling exponent $H \approx 0.54$ for 1 up to 85 days. For the DEM-USD, the situation is more complicated. For 32 to 512 quotes, one estimates a Hurst exponent $H \approx 0.45$, which seems to agree with the strong anti-correlation seen in the right part of Figure 2. Then, there seems to be a clear cross-over at about 512 quotes, after which one finds and exponent $H \approx 0.56$ between 512 to 8192 quotes. (In the average, 512 quotes corresponds to a physical time of 3 hours, and 8192 with approximately 2 days.) The value of the exponent $H=0.56$ is compatible with the value $H=0.59$ found in Ref. [15] for scales ranging from 2 hours up to 3 months.

When confronted with such results it is not always clear whether the observed deviations from the Gaussian behavior are significant. Therefore it is important to do additional analysis. For example, the value of $H=0.45$ is only possible when the DEM-USD time series is anticorrelated on scales less than 512 quote. On the other hand, the observed values of the Hurst exponent exceeding $1 / 2$ can either be due to long tailed distributions of returns, or due to long range positive correlations, or a combination of both. In cases where correlations are important, it is not necessary that the scaling behavior of the mean absolute lag- $k$ returns, extends to a distributional self-similarity as expressed by Equation 1. It is this aspect that we now discuss.

\section{Distributional self-similarity}

In order to analyze whether the distribution of the absolute returns is scale invariant, we apply Equation 1 as follows. We slightly modify it to reflect the scale invariance of the rates of return $(\rho)$, which we feel is financially more relevant, i.e.,

$$
\frac{Y(t+k)-Y(t)}{k} \text { i.d. }\left(\frac{k}{k^{\prime}}\right)^{H-1} \frac{Y\left(t+k^{\prime}\right)-Y(t)}{k^{\prime}} .
$$

We denote the probability density of these rates of returns over time periods of size $k$ by $P_{k}(\rho) d \rho$, that is, $P_{k}(\rho)$ is the probability density of the left hand side of Equation 2. Equation 2 implies for the densities that

$$
(H-1) \ln k+\ln P_{k}(\rho)=\ln Q\left(k^{1-H} \rho\right),
$$

that is, the process is distributional self-similar if plots of the densities $P_{k}(\rho)$ collapse onto the function $Q$, when plotting

$$
(H-1) \ln k+\ln P_{k}(\rho) \text { versus } k^{1-H} \rho .
$$

If this is the case, then the distribution of the returns on all scales $k$ is fully determined by the "basic distribution" $Q$, and the scaling exponent $H$.

An attempt to collapse the distributions of $k$-day rates of return using Equation 3 with $H=0.54$, is shown in Figure 4. It should be noted that the maximum of the various densities have been shifted vertically to 0 by force[11, 12]. The collapse is remarkable, and one finds that the estimated shape of the basic distribution for all 30 DAX stocks is asymmetric and has a distinctly convex left tail. For the DEM-USD high-frequency data the situation is more involved. 

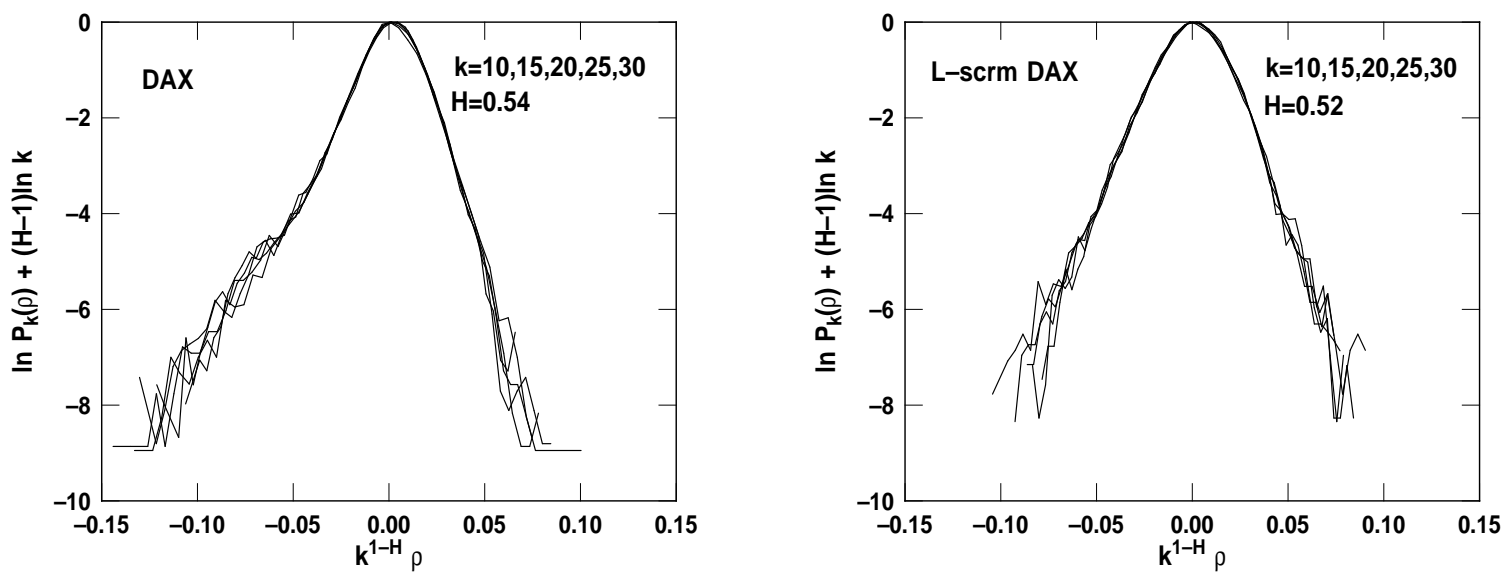

Figure 4: Left) The basic distribution $Q$ for the DAX stocks in the period November 3, 1986 till August 4, 1993. The rescaling rule Equation 3 is used with the self-similarity exponent $H=0.54$. Right) The same analysis, however, with DAX stocks in which the daily returns have been scrambled to get rid of correlations. The change in shape shows that correlations between the daily returns do play a role in the price formation. The tails are a bit shorter, because a shorter period has been used - November 3, 1986 till September 7, 1992.
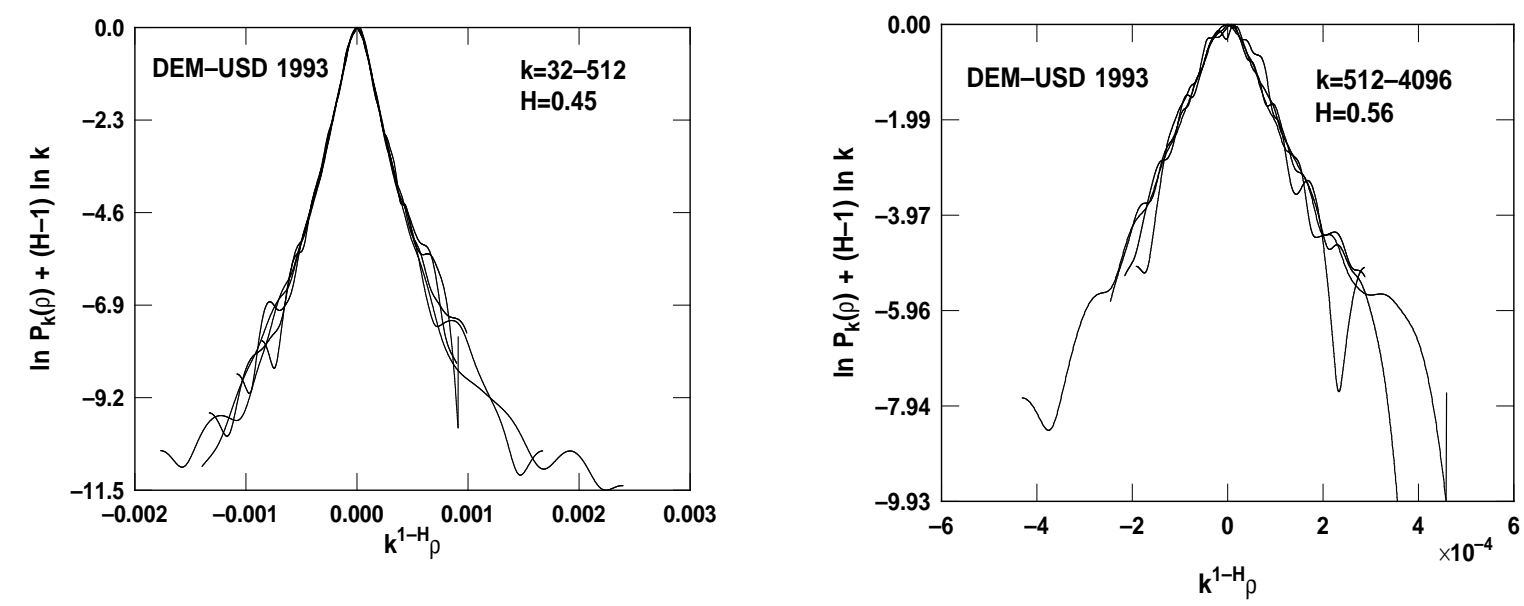

Figure 5: For the DEM-USD price record there is cross-over in the scaling behavior of the mean absolute lag- $k$ return. Left) For time scales of 32-512 quotes, one finds a high degree of distributional self-similarity, with the basic distribution $Q$ shown in the left plot. The right plot show the basic distribution for the longer time scales. One observes that not only the exponent $H$ changes when going from the short time scaling regime to the long time one: Also the shape of the basic distribution changes.

Because of the cross-over in the scaling behavior in the right part of Figure 3, the distributional self-similarity can only be expected to hold separately within the scaling regimes. The plots presented in Figure 5, show that also here there is a very high degree of distributional selfsimilarity in each of the scaling regimes, characterized by their corresponding Hurst exponents. 
Clearly, non of the basic distributions shown in Figures 4 and 5 is Gaussian. A Gaussian basic distribution would look like a graph of $-x^{2}$, which is symmetric with strongly concave tails. This certainly excludes fractional Brownian motions as very realistic models. However, like in fractional Brownian motions, correlations do play a role in the price records: doing the same analysis on the time-scrambled[12] version of DAX price records yields very different basic distributions, with a different self-similarity exponent $H=0.52$. For the 30 DAX stocks, this rescaling is shown in the right-hand part of Figure 4. After time-scrambling, which takes away all possible correlations in the price record, the basic distribution becomes symmetric and both tails become slightly concave. For the time-scrambled USD-DEM the basic distribution becomes Gaussian for all $k>32$, with self-similarity exponent $H=1 / 2$. What these effects of the time-scrambling on the estimated Hurst exponents and the resulting shapes of the basic distributions shows, is that correlations do play a very important role, at least on the short time scales considered here. This implies that one can also exclude i.i.d. Lévy stable models for the price increments on such scales.

Because the correlations play a crucial role in shaping the basic distributions, it seems the more remarkable that the price records analyzed here have such a large degree of distributional self-similarity. It implies that the observed self-similarity is not the result of any simple random process with stationary increments. This could mean that the market actively, and perhaps unknowingly, self-organizes so as to achieve this distributional self-similarity. In this respect it would be important to find the cause of the cross-over in the self-similarity observed at 512 quotes in the DEM-USD data. This cross-over means that the scaling behavior - Equation 2 with $H=0.56$ - that relates investors speculating on scales above $k=512$ quotes, can not be used to extrapolate to smaller time scales, which have their own scaling behavior with $H=0.45$.

\section{Implications}

The results discussed here have consequences for the evaluation of the risk of big losses or gains as a function of time, and thus for the evaluation of option prices. In all these cases it is important to estimate the probability of returns over different time lags. In a Gaussian market, the mean absolute rate of return decreases as $k^{-1 / 2}$ and the probability for a mean daily rate of return of $\rho \%$ over an $k$-day investment period obeys a large deviation principle[11, 12, 16, 17, 18, 19], and thus decays exponentially $\left(e^{k C(\rho)}, C(\rho)<0\right)$ as a function of $k$.

From Equation 2 and 3, it follows that the random variable $z=k^{1-H} \rho$, which is the scaleinvariant rescaling of the lag- $k$ rate of return, has probability density $Q(z)$, independent of the scale $k$. The shape of these densities is found in Figures 4 and 5 , but the functional forms are unknown. However, to illustrate the effect of these shapes on the behavior of the probabilities of returns over different time lags, we assume that the observed tails have functional forms such as $Q^{a}(z) \sim e^{-c z^{\nu}}$ or $Q^{b}(z) \sim z^{\lambda-1}$, with $\lambda>0$. With the exception of the right tail of the DAX in Figure 4, it is clear that values $0<\nu \leq 1$ are needed to reproduce the convexity of the tails and that the Gaussian value $\nu=2$ is thus excluded. A change of random variable $\left(z=k^{1-H} \rho\right)$ yields,

$$
\begin{aligned}
P_{k}^{a}(\rho) & \sim k^{1-H} e^{-k^{\nu(1-H)} c \rho^{\nu}} \\
P_{k}^{b}(\rho) & \sim k^{-\lambda(1-H)} \rho^{-\lambda-1}
\end{aligned}
$$




$$
P_{k}^{G}(\rho) \sim k^{\frac{1}{2}} e^{-k \rho^{2}} .
$$

Note that the Gaussian case, $P_{k}^{G}$, can be obtained from $P^{a}$ with $H=1 / 2$ and $\nu=2$, or from large deviation theory $[11,19]$. Making the conservative choice $\nu=1$, and using the value $H=0.55$ yields $P_{k}^{a}(\rho) \sim \exp \left\{-c k^{0.45} \rho\right\}$. Comparing this with what is expected in a Gaussian market, i.e., $P_{k}^{G}$ in Equation 5, one first notices that the probability for a large average rate of return $\rho$ [per unit time] over a period $k$, decays less fast $\left(\exp \left\{-c^{\prime} \rho\right\}\right)$ for the real market than for a Gaussian one $\left(\exp \left\{-c^{\prime} \rho^{2}\right\}\right)$ - note that the dependence of $c^{\prime}$ on $k$ has been suppressed to support the dependence on the return rate $\rho$.

Perhaps more important, one finds that the probability for a large rate of return $\rho$, decays like $\exp \left\{-c^{\prime \prime} k^{0.45}\right\}$ for the real market, versus $\exp \left\{-c^{\prime \prime} k\right\}$ for a Gaussian market, as a function of the holding period $k$-here we suppressed the dependence of $c^{\prime \prime}$ on the $\rho$ to stress the dependence on holding period $k$. That is, the probability for large returns or losses decays less fast for the real market than for Gaussian market. The situation is similar, even though a bit more pronounced, if a power-law fit, $P_{k}^{b}(\rho)$, is used instead of $P_{k}^{a}(\rho)$ : The probabilities decay as a powerlaw, both as a function of the rates of return $\rho$ and as a function of the holding time $k$.

Options pricing models like the Black-Scholes models[20], are heavily based the Gaussian assumption. In particular the shape and the scaling exponent $H=1 / 2$ are used. The distributional self-similarity discussed in this paper could be used for more realistic options pricing.

\section{Acknowledgment}

I would very much like to thank Heinz-Otto Peitgen for his support of this research. I am grateful to Wilhelm Berghorn, Kathrin Berkner and Michael Reincke for discussion. The data has been plotted using a graphics software package (VP) developed by Richard F. Voss.

\section{References}

[1] B.B. Mandelbrot: The fractal geometry of nature W.H.Freeman, San Francisco (1982)

[2] In right plot of Figure 1 (C) is the daily, (B) the weekly and (A) the monthly DAX record.

[3] L. Bachelier: Theory of Speculation thesis (1900) reprinted in Ref [4] 17-78

[4] P.H. Cootner: The random character of stock market prices The M.I.T. press, Cambridge, Massachusetts, (1964)

[5] B.B. Mandelbrot: The variation of certain speculative prices. The Journal of Business of the University of Chicago 36, 394-419 (1963)

[6] P. Mirowski, "Mandelbrot's economics after a quarter century" in Fractal geometry and analysis, eds. C.J.G. Evertsz, H.-O. Peitgen, R.F. Voss, Fractals fall edition and (World Scientific, 1995)

[7] B.V. Gnedenko and A.N. Kolmogorov, Limit distributions for sums of independent random variables Addison-Wesley (1968)

[8] W. Feller An introduction to probability theory and its applications Vol. 1, Wiley (1950)

[9] B.B. Mandelbrot and J.W. van Ness: Fractional Brownian motions, fractional noises and applications, SIAM Review 10, 4, 422-437 (1968) 
[10] B.B. Mandelbrot and J.R. Wallis: Computer experiments with fractional Gaussian noises I, II, III, Water resources research 5, 1,228-267 (1969)

[11] C.J.G. Evertsz and K. Berkner: Large deviation and self-similarity analysis of curves: DAX stock prices Chaos, Solitons \& Fractals 6 121-130 (1995)

[12] C.J.G. Evertsz: Self-similarity of high-frequency USD-DEM exchange rates, Proc. of first Int. Conf. on High Frequency Data in Finance, Zurich 1995, Vol. 3. (1995)

[13] The High Frequency Data in Finance 1993 data set have been obtained from Olsen \& Associates "Research institute for applied Economics", e-mail:hfdf@olsen.ch

[14] R.F. Voss: 1/f noise and fractals in Economic time series In: Fractal geometry and computer graphics J.L. Encarnação, H.-O. Peitgen, G. Sakas, G. Englert (Eds.) SpringerVerlag, 45-52 (1992)

[15] U.A. Müller, M.M. Dacorogna, R.B. Olsen, O.V. Pictet, M. Schwarz, C. Morgenegg: Statistical study of foreign exchange rates, empirical evidence of price change scaling law, and intra-day analysis, Journal of Banking and Finance 14, 1189-1208 (1990)

[16] B.B. Mandelbrot: An introduction to multifractal distribution functions, in Random fluctuations and pattern growth H.E. Stanley and N. Ostrowsky, Kluwer Academic Publishers, Dordrecht, 279-291 (1988)

[17] C.J.G. Evertsz and B.B. Mandelbrot: Multifractal measures in Ref [18], 921-953 (1992)

[18] H.-O. Peitgen, H. Jürgens, D. Saupe: Chaos and Fractals Springer-Verlag, New York, (1992)

[19] J.A. Buckley: Large deviation techniques in decision, simulation and estimation Wiley (1990)

[20] T.J. Watsham Options and futures in international portofolio management Chapman \& hall (1992) 